\title{
Convolutas
}

\section{Developable strips and digital fabricated lightweight architecture}

\author{
Francisco Gonzalez-Quintial ${ }^{1}$, Andres Martin-Pastor ${ }^{2}$ \\ ${ }^{1}$ ETSA AGET University of the Basque Country ${ }^{2}$ ETSIE University of Seville \\ Ifrancisco.gonzalez@ehu.eus ${ }^{2}$ archiamp@us.es
}

The present research is mainly focused on the development of a system that would be able to offer the potentiality of constructing free-form surfaces by using developable surfaces. Through a deep revision of pre-computational traditional geometric systems based on the classical Descriptive Geometry, after a re-interpretation that design and algorithmic generation tools allow by using computers and digital fabrication hardware as testing ground, a geometric control process has been designed in order to offer the possibility of managing double-curvature complex forms and their adaptation by using developable surfaces. The focal point of this system is proving how developable surfaces are suitable to build architectonical elements at real scale.

Keywords: Geometry, Developable Surfaces, Algorithmic Approach, Digital Fabrication

\section{INTRODUCTION}

This research is mainly focused on the development of a complete design system where geometric control, scale prototypes fabrication and big scale physical fabrication would be embedded and interrelated, being tested as experimental pavilions. In this way, the whole process has been closed starting from the basic computer aided design to the fabrication of final functional elements by means of digital fabrication tools, which allows a complete control throughout the whole process.

The construction of free-form surfaces is restricted to what is possible by means of graphical and constructive control. Regarding this subject, the adaptation of free forms through the use of developable surfaces by using different geometric systems has been an important line of research in recent years. Although nowadays there have been different important approaches (Shelden et al. 2002, Glymph et. al. 2004, Spuybroek 2004, Pottman et al. 2007, Piker) we have followed some issues that come directly from the Classical Descriptive Geometry Theory (Monge 1850) jumping from physical to digital drawing systems that nowadays the use of certain CAD software allows. The approach came from the enhanced graphic thinking via-Geometry based on Graphics-(Martín-Pastor 2019) pass through the common digital software and hardware tools and reach physical developments where the systematization of these theoretical developments actually prove their constructive validity.

The main objective of this research consist of finding results that can be extrapolated from the theoretic geometrical realm into the physical and 


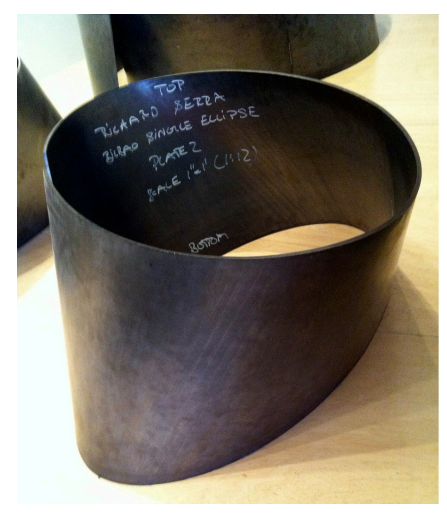

constructive environment, maintaining the design and fabrication processes under exhaustive geometric control through all over the process. For that purpose, several types of prototypes and small pavilions have been fabricated using cardboard and small thickness plywood panels. This material is quite suitable and fast to be machined by means of a milling or laser-cutting machine tool at almost any digital fabrication laboratory all over the world.

\section{GEOMETRIC BASIS AND METHODOLOGY STATEMENT}

Overall, the movement of a plane through the space generates a developable surface. In order to obtain this kind of surfaces we have to introduce some constraints to this movement. Specifically, one of the ways we can obtain a developable surface is by sliding a plane tangentially leaning along any pair of curves. Whether they could be planar or warped curves, this plane is tangent to these two curves at least at one point along each one. The line that joins this pair of points will be considered as a generatrix of the developable surface that the moving plane generates. Some authors have referred to these kinds of developable surfaces or developable strips with the specific name of convolutas (Asensi, 1999). In some treatises about Classical Geometry these processes have been developed manually by using auxiliar graphical systems (Taibo, 1983), the graphical

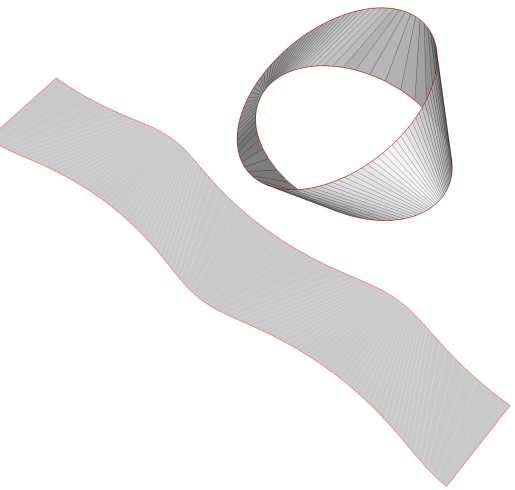

Figure 1

Graphic

development of

Torqued Ellipse by

Richard Serra.

(Image and

Drawing by the authors) manual layout of these kind of surfaces is not in the habit of raising difficulty when at least one of the curves is planar. The process turns out much more complex when both curves are warped and in the case of free curves, the system appears unapproachable without the support of CAD software.

This type of planar or developable elements is used because of the simplification and economy that it offers regarding to economic and constructive aspects. A developable surface can be constructed from a planar surface, and the other way round a developable surface can be deployed on a plane without any crease or distortion. This question is decisive in manufacturing complex surfaces within any kind of constructive system, whether it would be a front facade panel or the hull of a ship. From the geometric point of view, many aspects define a developable surface. One of the most determining is its Gaussian curvature, which at any point over a developable surface is characterized by having always zero value.

Because of that and despite of the complexity concerned to this special kind of surfaces the practical use of them is widespread among not only engineering, architecture and naval construction but there are also some remarkable sculptural works (figure 1) that are based on this geometric system. (Koman, Serra)

In order to systematize the design process, some different approaches and algorithms has been generated, refined and customized following and rewriting 
Figure 2

Developable tangent surface.

Concept.

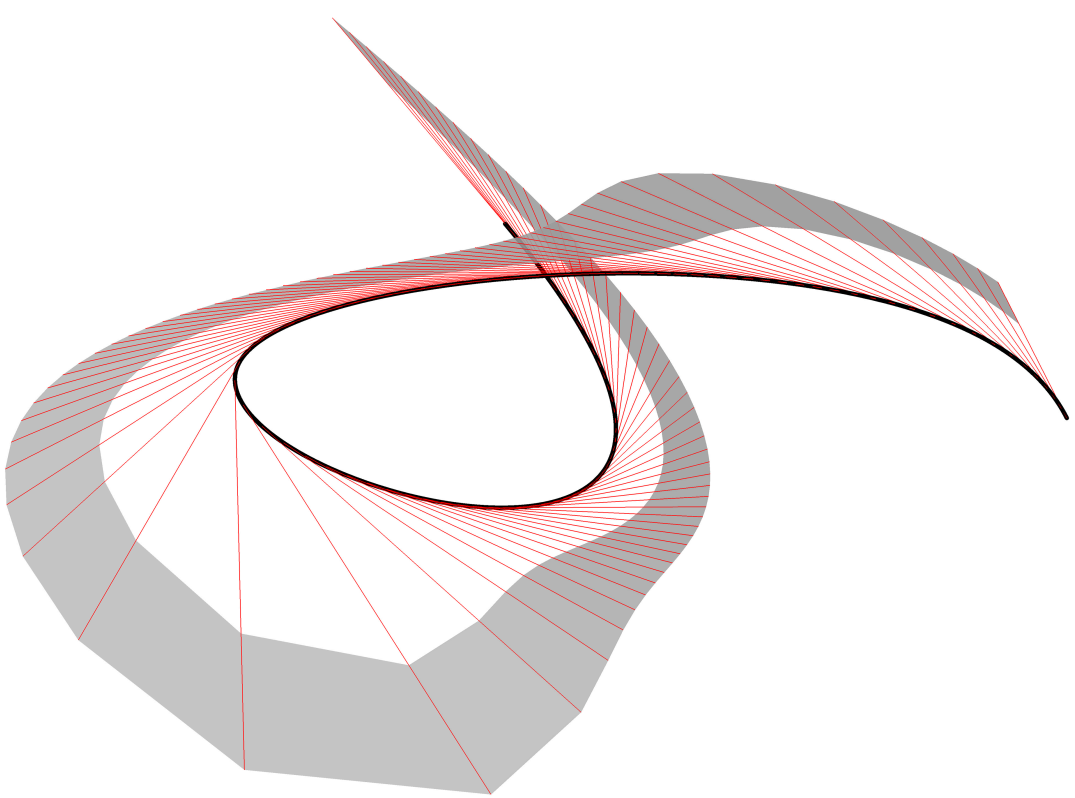

the common theoretical mechanism referred above by using parametric tools, specifically Grasshopper plugin for Rhinoceros. These approaches allow the application of the system by using any pair of spatial curves that would be defined; these should always be constrained among some specific intrinsic geometric parameters that reduce somehow its scope. (Quintial et al. 2012) The refinement of these processes have resulted in the theoretical concept and the geometric definition that is described below and it was used to generate the constructive elements that are hereinafter described in this paper.

The developable tangent surface (figure 2) is generated by transition of the different tangent lines, which are traced to a curve. From the pure geometrical point of view is the only developable surface that can be obtained by using a line or vector directly associated to a curve in different way that the general system previously proposes by meaning the movement of a plane through the space.
Given two warped curves it can be easily traced their corresponding tangent developable surfaces. These two surfaces could have an intersection along another warped spatial curve - it would be possible only if they do not belong to parallel planes, in this particular case they would have an improper intersection at the infinite -. From each point the intersection curve will be divided, in this way several pairs of straight lines can be traced from these points that would be tangents to the original warped curves, namely taking any point along the intersection curve would be a pair of straight lines that cutting at this point will be tangents to both original curves. The latter two tangent straight lines actually are secant one to each other therefore they determine, as could not be otherwise, a tangent plane to both curves.

The segment of line that is obtained by the tangent point at the curves constitutes a generatrix of the developable surface. Moreover, the developable strip that rests on both curves is achieved by the tran- 


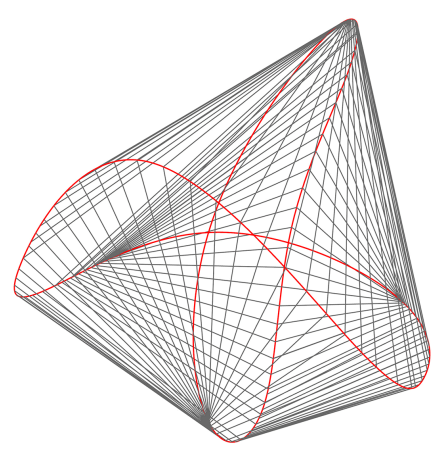

sition of several segments. (figure 3) Hereinafter we will name this developable strip as convoluta in the way is named in the previous referred literacy.

The graphic statement described above is affordable by using computational graphic tools, as in our particular case the plugin Grasshopper. The process turns out quite simple to be arranged into a logic definition just by following the steps that are described. The most important theoretical issue is related to the fact that all the possible solutions to the problem focused on obtaining developable stripes from pairs of curves supposes, is finally defined and delimited by determining the geometric place of the points that both tangential developable surfaces share.

At this point is interesting to highlight the point that both tangential surfaces are developable surfaces and the direction of the tangent vector coincides with one of the principal curvature directions at the point on the surfaces. In this special case, this one which counts with zero value of gaussian curvature. In other words, that direction is the same that the tangent to the osculator circle of infinite radius. If we think on the developable surface as a parametric one we can easily compute both principal directions as $u-v$ isoparametric curves. This way of proceeding simplifies absolutely the difficulties that represent the question of dealing with tangents to the curves that represent an unfulfilling question in our previous approaches.

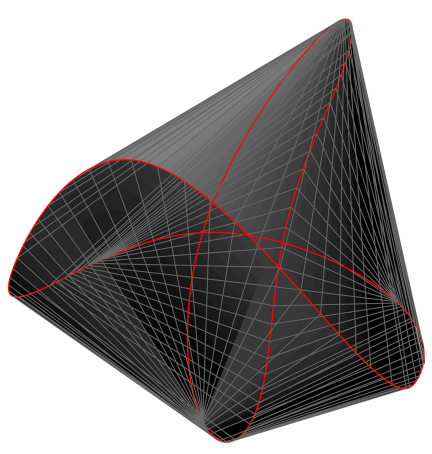

Figure 3

Convoluta obtained

from two warp

closed curves
From the geometrical point of view, the weakness of the system is closely related with the relationship between both curves and their relative curvature. It means that there are some unavoidable geometric constraints that establish the limits to the geometric developments.Then the main objective of tracing developable strips suitable to be fabricated is imposed by the pure geometric reality and we can affirm that along some special zones absolutely developable strips from the geometric point of view are impossible to be obtained. From this point, it is necessary some kinds of adaptative approaches to confer construct validity to the theoretical proposal. The delimitation of this zones and characteristics of the curves and its relationship as well as the way to solve this special cases and discontinuities are subject to continuous approaches and will be considered in future works.

\section{RESULTS}

As it has been presented above, The main focus of this theoretic development follows a practical objective that can be materialized in Architectonic constructions. In this way, several physical models have been built in order to validate the processes. Some of them finished only at prototype scale (figure 4) and other ones at real scale by means of different small pavilions. 
Figure 4

Small prototypes of free form surfaces adapted by using convolutas

Figure 5

Cactus pavilion.

Santiago of Anaya, Mezquital Valley,

Mexico.

Convolutas,

Developable Strips made of $3 \mathrm{~mm}$.

plywood.
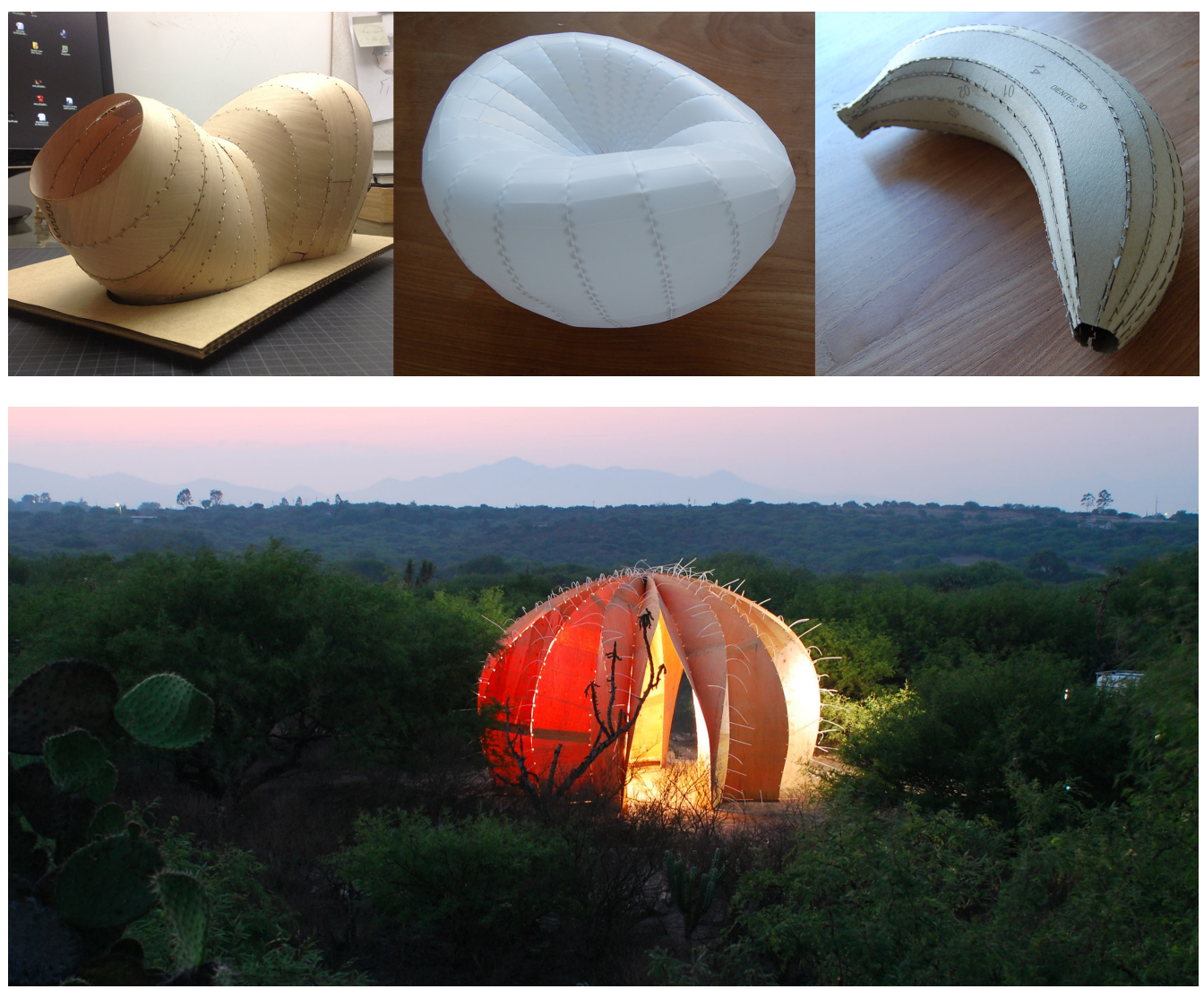

\section{Cactus pavilion}

The project is defined as a double-sided statement. On the one hand, it presents a real constructive experience regarding experimental lightweight Architecture. This experience involves in-depth research around Advanced Geometry, Digital Fabrication and ephemeral architecture applied to heritage, along the lines of low-cost construction and environmental concerns. On the other hand, the proposal advances in the exploration of adaptive envelopes, reactive materials, and the continuity of geometric development towards the use of developable and re- sponsive surfaces in Architecture.

The Cactus Pavilion (figure 5) is a real wooden pavilion developed as roving interpretation centre of the Architectural \& Natural Heritage Rescue Project of Santiago de Anaya, Mexico.

The pavilion is a stand-alone architectural object that, by itself, strives to represent the values of the project and its connection with the landscape and the environment. The shape of a cactus was proposed as a biomimicry architectural form, inspired by an endemic species of this valley, Echinocactus plathyacanthus, and its 'cristata' forms. 


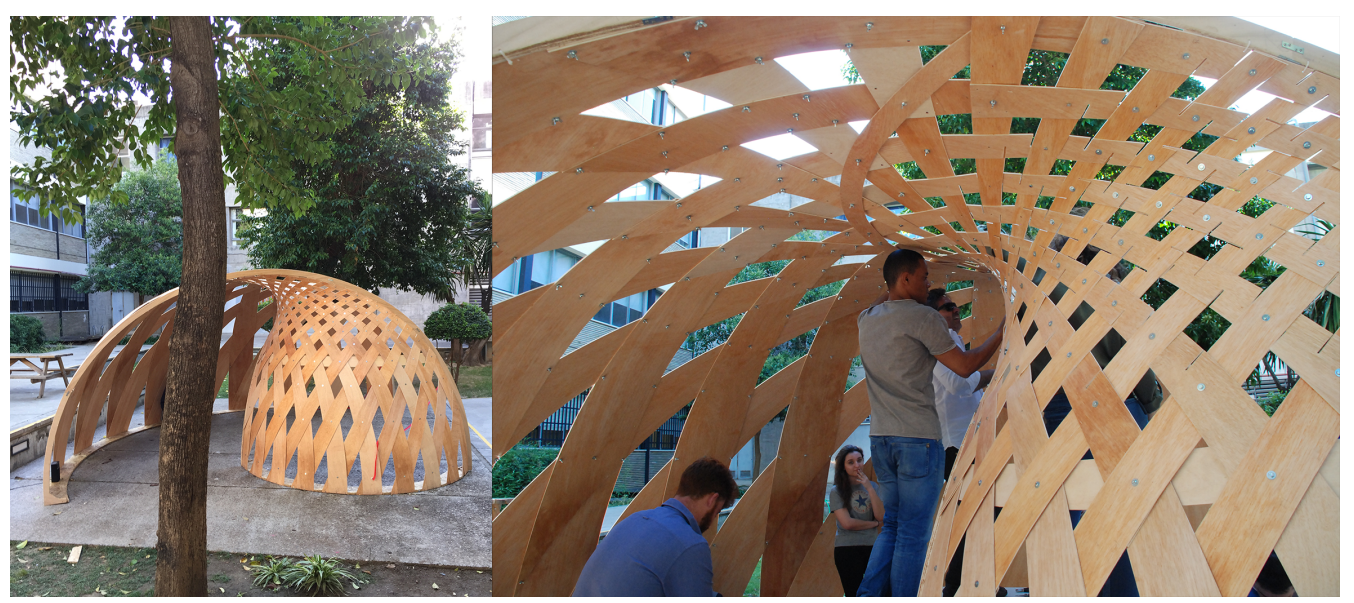

The installation uses natural materials and a sustainable manufacturing model, which has been developed as a lightweight construction, with an easy assembly process. Likewise, the architectural installation is innocuous where environmental protection is concerned, leaving no traces or residues in the placements. The pavilion was designed by employing CADCAM and parametric tools and was mechanized by using a common CNC milling machine. The architectural installation is made of 4-mm-thick plywood strips that, once arranged, bent, and joined together with nylon cable ties, forms a self-supporting structure. The design, construction and assembling of this pavilion has been carried in diferent spaces all over the world, Universidad de Sevilla, Spain; Universidad del País Vasco, Spain; and UNAM, Mexico. This circumstance has led to arrange a methodology that also bears in mind the idea of global design system while the materialization of the ideas remains locally. In this way, we have taken advantage of the possibility that increasingly global knowledge transmission offers nowadays.

The proyect has been designed by Department of Graphics Engineering, ETSIE, of Universidad de Sevilla (Andrés Martín Pastor); in collaboration with FabLab Donostia EHU-UPV Donostia, Spain
(Francisco González Quintial). Cactus Pavilion was awarded with a Honorable mention at __Laka-React Competition 2019, where complete credits can be consulted.

\section{Globoide Pavilion}

Globoide Pavilion is an experimental pavilion (figure 6), the result of the Teaching Innovation Project entitled: "Geometry and creativity. Design, Manufacturing and Assembly processes of Experimental architectures in University Education". This project involved Universidad del País Vasco (Fablab Donostia), Universidad del Litoral, Argentina; Universidad de la República (Uruguay); and it was conceived and coordinated by Department of Graphics Engineering, ETSIE, of Universidad de Sevilla. The general proposal consists of approaching the phases of design, digital fabrication, and assembly of a wooden pavilion by means of Descriptive Geometry and with digital tools. The academic project is articulated applying a theoretical-experimental model that is verified through the realization of small-scale prototypes and, finally, with the assembly of the full-scale architectural installation. This experience its an advance of previous experiences carried in the context of international workshop (Martín-Pastor, García Al-
Figure 6

Globoide pavilion.

ETSIE Sevilla.

Revolution of a

circle non-coplanar with the axis adapted by using convolutas. 
Figure 7

Parametric model. Obtention of the developable strips following imposed edges. The

Gaussian curvature analysis shows the developability of these strips.
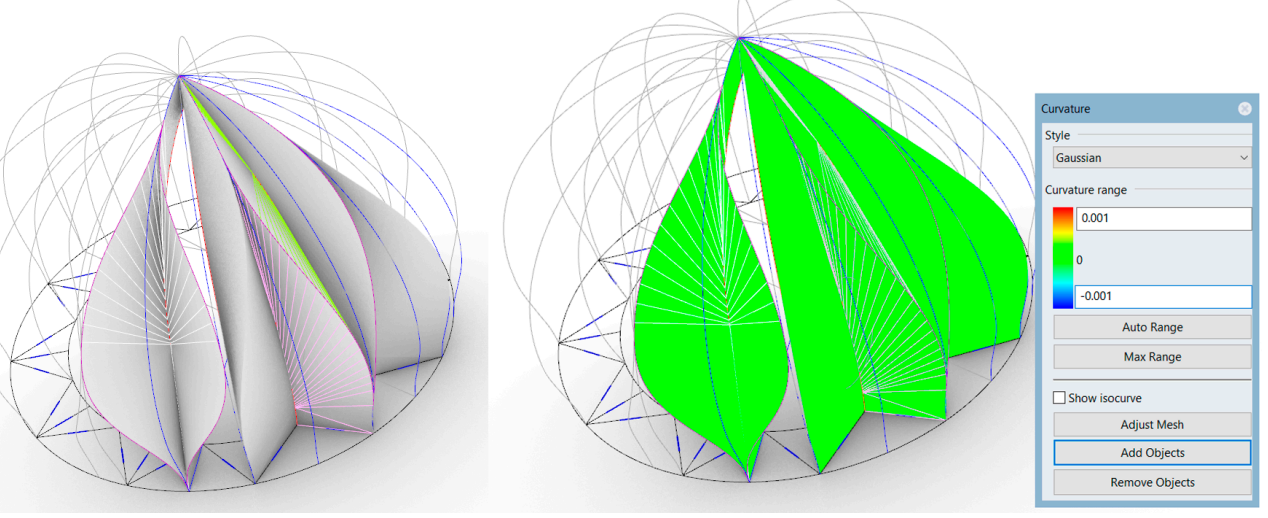

varado 2019)

The shape of The Globoide is generated by the revolution of a circle around an axis that should be non-coplanar, otherwise if both of them are co-planar the revolution surface generated is well known as thorus. The research objective of this project is focused on the evaluation of the system on this kind of special shapes where the gaussian curvature varies from one part to another. Actually constitutes more an approach to the adaptation of double curvature surfaces by using devolopable strips (convolutas) than an adaptation proccess to developable forms between a pair of curvas. Nevertheless, the process employed in this case is strictly the same with slightly different nuances. In this case the input values are circles on the thoroidal surface and the scope of the method, as has been signed above, reach some difficulties around the zone where the two curves cross. Purely, from the geometric point of view, the concept that is embedded in the core of these cases is based on the use of Convolutas following the development exposed above. (figure 7) In this way an absolute parametric model was developed where the curves that define the edges of the strips were well defined and at the same time they could be easily adapted by varying slightly the parameters that define them. Progressive approaches to the form allow to deal with the form of tracing the appropriate forms of the strips in order to be fabricated.

Purely, from the geometric point of view, the concept that is embedded in the core of these cases is based on the use of Convolutas following the development exposed above. (figure 7) In this way an absolute parametric model was developed where the curves that define the edges of the strips were well defined and at the same time they could be easily adapted by varying slightly the parameters that define them. Progressive approaches to the form allow to deal with the form of tracing the appropriate forms of the strips in order to be fabricated.

Before affording the real construction, at lower level we proceeded to physically check the suitability of the system, the accuracy of the designing results and the behavior of the material we are planning to use by constructing proportional prototypes at minor scale. (figure 8) In this way, the differences between the prototype and the real building can be foreseen and the troubleshooting can be anticipated as far as construction issues are concerned.

This phase of the work is also based on the structural analysis of the material and its behavior like bending active strips by using parametric structural analysis software, in this case Karamba (figure 9), which is a Finite Elements Method modeler that al- 


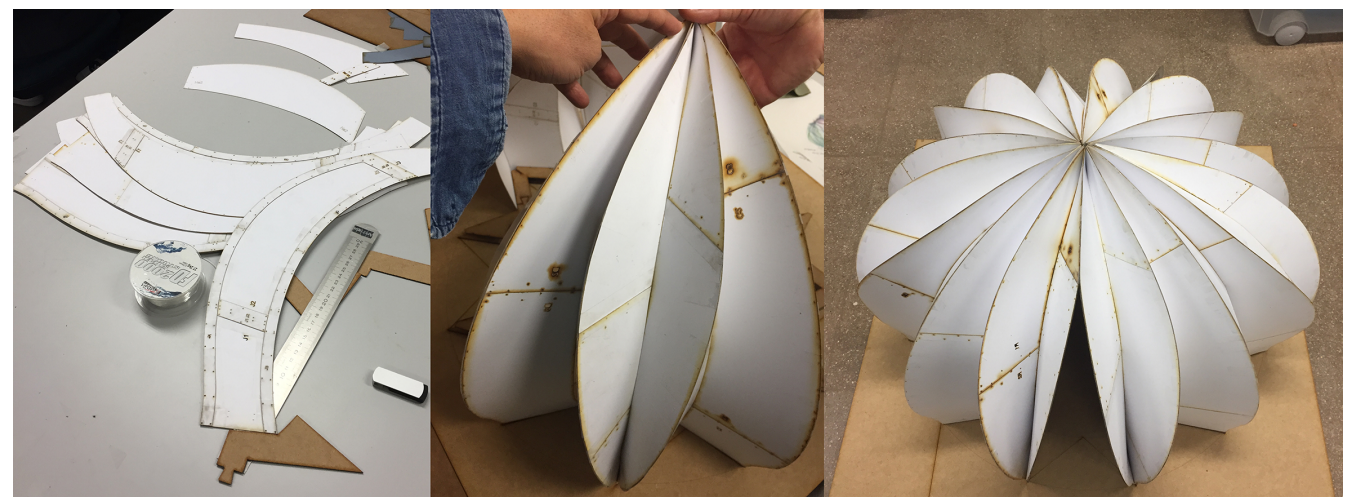

Figure 8

Prototype made of cardboard at 1:5

scale

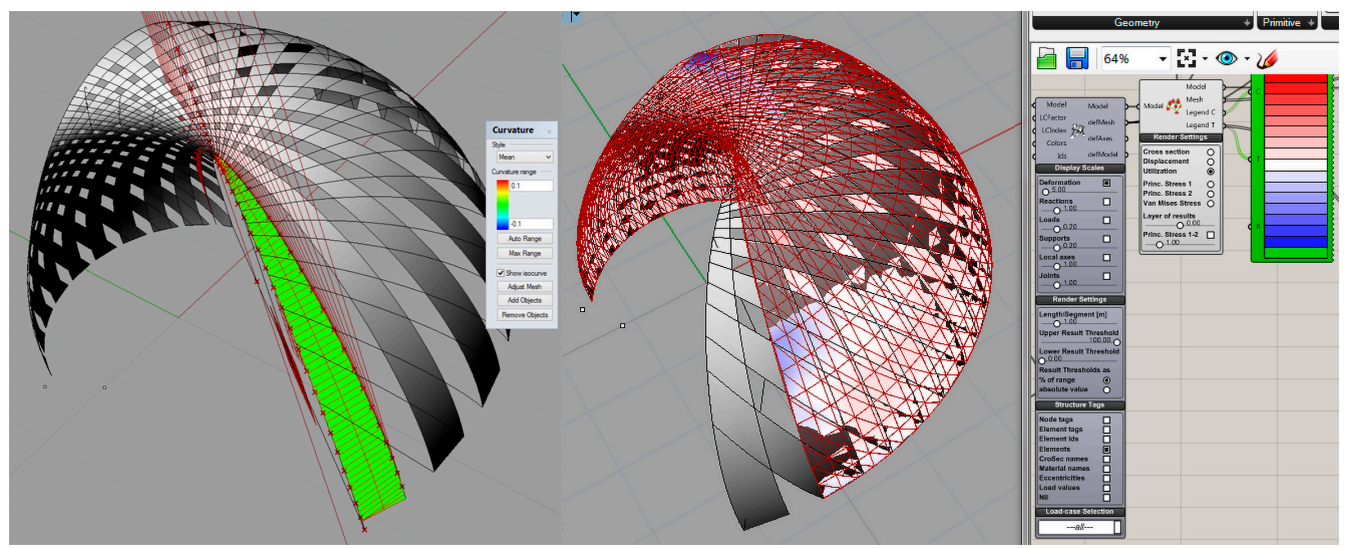

Figure 9

Geometric and

structural analysis

of the surface. 
lows the graphic analysis into the parametric model. In this way, all the process is developed inside the same graphical environment and is produced by using the same chain of algorithms written.

\section{CONCLUSION}

The application of the method demonstrates to be satisfactory in the analyzed cases. The systematizing of a process formulated in the classic theory of the Descriptive Geometry -Geometry from the Graphicby means of a new algorithmic process allows to validate first the theoretical general method and to allow its application in such a way that was turning out to be impossible before the generalized use of digital tools.

As is commonly know, the use of developable surfaces to model free surfaces or double curvature surfaces has an important application in different areas and specially in Architecture so it supposes, apart from other interesting mathematical approaches, a suitable development considering it allows the use of the traditional materials without the need to use much more expensive curved processes.

Though we are working at the jump from the prototype to the real construction elements, nowadays this constitutes one of the most exciting future ways of research. Moreover we have to bear in mind that we are working with CAD software and simultaneously more and more we are getting used to digital fabrication hardware as 3D printers, laser cutting and CNC machines.

We speak about prototypes at major or minor scale, not models, in the prototype we can already anticipate and foresee the behavior of materials in relation to the way they are arranged and assembled. The prototype has a value in relation to purely formal model, we have seen the results in terms of tolerances, movement, adaptability of certain light materials and conclusions difficult to predict theoretically.

Regarding geometric concepts of the system we can indicate that new routes appeared are opened in the research and adaptation of the theory used in reference to the developable surfaces where we are mainly focused. The extension of this paper shall prevent the exposure of the entire system, there are located some new directions and spaces to enhance the system, overall in determining the gaps of the system. The exhibition of the rest of results due to this research line will be matter of future articles.

Finally we can add that we are profusely using and teaching the obtained developments in Advanced Geometry through the academic community by this connections between virtual and physical realms. In this way we can match the theoretical knowledge with the physical manufacturing and construction of architectonic elements through the use of new tools framed into the 4th Industrial Revolution.

\section{REFERENCES}

Glymph, J., Shelden, D., Ceccato, C., Mussel, J. and Schober, H. 2004, 'A parametric strategy for freeform glass structures using quadrilateral planar facets', in Skibniewski, M. J. (eds) 2004, Automation in Construction, Elsevier, pp. 187-202

Gonzalez-Quintial, F. 2013 'Freeform surfaces adaptation through developable surfaces using apparent contours', CAAD Futures 2013, Shangai

Gonzalez-Quintial, F. 2014 'Convolutas - Piece-wise Developable Surfaces using two Curved Guidelines', Annual International Conference on Architecture and Civil Engineering

Izquierdo-Asensi, F. (eds) 1999, Geometría Descriptiva Superiory Aplicada., Paraninfo, Madrid

Martin-Pastor, A. and Garcia-Alvarado, R. 2019, 'Developable wooden surfaces for lightweight architecture. Bio-Dune Pavilion., in Bianconi, F. and Filipucci, M. (eds) 2019, Innovative techniques of representation in architectural design. Lecture Notes in Civil Engineering., Springer

Martin-Pastor, A. 2019, 'Augmented Graphic Thinking in Geometry. Developable Architectural Surfaces in Experimental Pavilions', in Marcos, C. L. (eds) 2019, EGA 2018: Graphic Imprints, Springer, pp. 1065-1075

Monge, G. (eds) 1850, Application de I, Bachelier, Imprimeur Librarie, Paris

Pottmann, H., Asperl, A., Hofer, M. and Kilian, A. 2007, Architectural Geometry, Bentley I. Press. , Exton

Shelden, D. 2002, Digital Surface Representation and the Constructability of Gehry's Architecture., Ph.D. Thesis, 
Massachusetts Institute of Technology. Dept. of Architecture.

Spuybroek, L. (eds) 2004, NOX, Thames\&Hudson, London

Taibo-Fernandez, A. (eds) 1983, Geometría Descriptiva y sus aplicaciones. Tomo Il., Tebar Flores., Madrid

[1] https://lakareacts.com/winners/cactus-pavilion/

[2] http://kangaroo3d.com/

[3] http://www.koman.org/

[4] https://www.guggenheim.org/artwork/17143

[5] http://www.es.rhino3d.com/

[6] http://www.grasshopper3d.com/

[7] https://www.karamba3d.com 